\title{
Hochintensiver fokussierter Ultraschall (HIFU)
}

\section{G-BA bestätigt Effizienz bei Behandlung von Bauchspeicheldrüsenkrebs}

Berlin - In einem aktuellen Beschluss entschied der Gemeinsame Bundesausschuss (G-BA), dass der ultraschall-gesteuerte hochintensive fokussierte Ultraschall (HIFU) als Behandlungsmethode bei nicht chirurgisch behandelbaren bösartigen Neubildungen des Bauchspeicheldrüsenkrebses (Pankreas) eingesetzt werden kann. Die Deutsche Gesellschaft für Ultraschall in der Medizin e. V. (DEGUM) begrüßt diese Entscheidung - denn HIFU ist für diese Patienten eine effektive und oftmals die letzte Methode zur Schmerzlinderung, wenn Medikamente versagen. Auch das Tumorvolumen kann bei vielen Patienten durch diese Ultraschall-Anwendung reduziert werden. Auf völliges Unverständnis seitens der DEGUM stößt jedoch die Ablehnung des G-BA, den Stellenwert des HIFU bei anderen Indikationen wie Leber-, Knochenmark- und Knochentumoren durch Studien zu evaluieren.

Die Fünf-Jahres-Überlebensrate bei Bauchspeicheldrüsenkrebs beträgt etwa $7-8$ Prozent. In den meisten Fällen kann der Patient nur noch palliativ behandelt werden. „Bei etwa 80 Prozent der Betroffenen ist die Lebensqualität durch den Tumorschmerz deutlich bis massiv eingeschränkt“, betont Professor Dr. med. Holger Strunk, Oberarzt der Radiologischen Universitätsklinik Bonn. „Im Verlauf der Erkrankung werden diese Schmerzen häufig sogar therapieresistent. Den Patienten hilft dann keine Schmerzmedikation mehr." Untersuchungen aus Deutschland und dem ostasiatischen Raum zeigen, dass der Einsatz von HIFU in etwa 80 Prozent der Fälle zur Schmerzlinderung bei den Betroffenen führt. „Wir begrüßen daher die G-BA-Entscheidung, dass HIFU bei diesen Patienten eingesetzt werden kann“, so Strunk.
Auch das Tumorvolumen kann durch diese Behandlung bei etwa zwei Drittel der Patienten signifikant verkleinert werden. Experten vermuten, dass HIFU nicht nur einen symptomatischen Nutzen hat, sondern dass diese Methode möglicherweise auch das Überleben positiv beeinflusst. „Hierzu fehlen uns noch weitere klinische Untersuchungen; doch erste Ergebnisse zeigen, dass HIFU ein großes Potential in der Tumorentfernung haben könnte“, erklärt Strunk. Diese Ultraschall-Technik bietet Anwendern und Patienten viele Vorteile: Die Behandlung erfolgt wenig invasiv und ruft dadurch in der Regel nur geringe Nebenwirkungen hervor.

Im Gegensatz zum diagnostischen Ultraschall bündelt der Ultraschallkopf beim HIFU die hochenergetischen Schallwellen wie bei einem Hohl-spiegel in einem Brennpunkt innerhalb des menschlichen Körpers. Durch die Absorption der Ultraschallwellen entsteht Wärme, die das Gewebe im Fokus des HIFU auf bis zu 80 Grad erhitzt. Bei diesen Temperaturen gerinnt das Eiweiß und die Zellen des Tumors sterben ab.

„Ein weiterer großer Vorteil ist, dass sehr präzise gearbeitet werden kann und auch Tumoren in der Nähe von Blutgefäßen behandelt werden können“, ergänzt Strunk. „Da keine Nadeln, Sonden oder Ähnliches eingebracht werden müssen, besteht zudem keine Gefahr einer Blutung oder der Tumorzellverschleppung, wie sie bei anderen Verfahren durch den Stichkanal vorkommen kann.“

Der G-BA hat auch überprüft, ob Studien vorliegen, die den Stellenwert des HIFU bei anderen Erkrankungen belegen. Da dies aber ein relativ neues Verfahren ist, gibt es bisher nur zu wenigen Krankheitsbildern
Studien, wie eben zum Pankreaskarzinom, dem Leberzellkarzinom und dem Gebärmuttermyom. Nichtsdestotrotz könnten zukünftige Untersuchungen auch hier weitere Indikationen aufzeigen. Auf völliges Unverständnis seitens der DEGUM stößt daher die Ablehnung des G-BA, den Stellenwert des HIFU bei anderen Indikationen wie Leber-, Knochenmark- und Knochentumoren oder der Endometriose, einer gutartigen Wucherung von Gewebe der Gebärmutterschleimhaut, durch Studien zu evaluieren.

„Mag ja sein, dass zukünftige Studien aufzeigen, dass es hier für den HIFU keinen Stellenwert gibt. Doch viele Patienten mit diesen Erkrankungen profitieren nachweislich von einem Einsatz des HIFU. Gerade bei der palliativen Behandlung von Knochenmetastasen am peripheren Skelett könnte HIFU zunehmend an Bedeutung gewinnen“, erklärt Strunk. „Nur weil derzeit keine deutschen Studien vorliegen, kann man doch nicht a priori weitere Studien ausschließen. Was ist das für ein Verständnis von Wissenschaft. Hier müssten unbedingt weitere klinische Studien zur Untersuchung des langfristigen Nutzens dieser Methode erfolgen“, so der DEGUM-Experte.

\section{Quellen:}

https://www.bibliomedmanager.de/nachrichten/politik/detailansicht/34463g-ba-krankenkassen-contra-patienten/ https://www.ncbi.nlm.nih.gov/pmc/ articles/PMC5376281/ https://www.aerzteblatt.de/archiv/ 186344/Hochintensiver-fokussierter-Ultraschall-HIFU-(1)-Erste-standardisierte-Studien-laufen 